\title{
Conformal entropy from horizon states: Solodukhin's method for spherical, toroidal, and hyperbolic black holes in $D$-dimensional anti-de Sitter spacetimes
}

\author{
Gonçalo A. S. Dias and José P. S. Lemos \\ Centro Multidisciplinar de Astrofísica - CENTRA \\ Departamento de Física, \\ Instituto Superior Técnico, \\ Universidade Técnica de Lisboa, \\ Av. Rovisco Pais 1, \\ 1049-001 Lisboa, Portugal
}

(Dated: September 22, 2018)

\begin{abstract}
A calculation of the entropy of static, electrically charged, black holes with spherical, toroidal, and hyperbolic compact and oriented horizons, in $D$ spacetime dimensions, is performed. These black holes live in an anti-de Sitter spacetime, i.e., a spacetime with negative cosmological constant. To find the entropy, the approach developed by Solodukhin is followed. The method consists in a redefinition of the variables in the metric, by considering the radial coordinate as a scalar field. Then one performs a $2+(D-2)$ dimensional reduction, where the $(D-2)$ dimensions are in the angular coordinates, obtaining a 2-dimensional effective scalar field theory. This theory is a conformal theory in an infinitesimally small vicinity of the horizon. The corresponding conformal symmetry will then have conserved charges, associated with its infinitesimal conformal generators, which will generate a classical Poisson algebra of the Virasoro type. Shifting the charges and replacing Poisson brackets by commutators, one recovers the usual form of the Virasoro algebra, obtaining thus the level zero conserved charge eigenvalue $L_{0}$, and a nonzero central charge $c$. The entropy is then obtained via the Cardy formula.

PACS numbers: 04.70.Dy, 11.25.Hf, 04.60.-m, 04.50. $+\mathrm{h}$
\end{abstract}




\section{INTRODUCTION}

The Bekenstein-Hawking formula for the entropy $S$ of a Schwarzschild black hole [1, 2], given by

$$
S=\frac{A}{4 G}\left(\frac{k_{\mathrm{B}} c^{3}}{\hbar}\right),
$$

being directly proportional to the 2-dimensional horizon area $A$ and inversely proportional to the constant of gravitation $G$, assigns to the black hole a gravito-geometric entropy. This entropy, together with the corresponding Hawking temperature $T=\hbar c^{3} /\left(8 \pi k_{\mathrm{B}} G M\right)$ [2], where $M$ is the black hole's mass, has brought the black hole into the frame of thermodynamics (see, e.g. [3]). Thermodynamics is a phenomenological theory, which ignores in general the fundamental constituents, or degrees of freedom, of the object in consideration. On the other hand, statistical mechanics, together with the corresponding degrees of freedom, provides a much more fundamental way of understanding the phenomena. Thus, to have a better understanding of the degrees of freedom that give rise to this gravito-geometric entropy one should resort to statistical mechanics. Now, classically a black hole is characterized by its mass $M$, charge $Q$, and angular momentum $J$, less than a handful of degrees of freedom. This characterizes the no-hair of black holes, i.e., the non existence of classical degrees of freedom. So where should one look for hairs? The dependence of the black hole entropy on $\hbar^{-1}$ in Eq. (11), suggests that the hairs, or the degrees of freedom, are essentially quantum mechanical. But what are they, and where are they located?

These are problems that so far have no definite answers. The black hole entropy could come from microscopic matter degrees of freedom induced by the quantum atmosphere of the matter fields generated by the Hawking radiation, in which case the degrees of freedom should be located in the horizon neighborhood, as has been advocated by several authors (see, e.g., [4] for a review). On the other hand, since the whole phenomenon is gravitational in nature, one can be led naturally to advocate the very existence of gravitational degrees of freedom that give rise to the gravito-geometric entropy $S$, given in Eq. (II). This idea was first raised by Bekenstein who speculated early that the degrees of freedom should be related to the quantization of the black hole area, emphasizing thus that they are in the gravitational field [5].

Theories that advocate to be quantum theories of gravity, such as string theory, loop quantum gravity, and induced gravity, have provided important calculations that give the 
entropy as in Eq. (11). However, neither of the two questions raised above have a concrete answer. For instance, string theory, with its attached D-brane technology, has been successful in dealing with extreme black hole horizons, but not with non-extreme horizons or cosmological horizons (such as de Sitter). Moreover, the celebrated Strominger and Vafa D-brane calculation [6] is done in a regime for which the black hole is, in practical terms, a point like object from a distance, and not in a regime for which there is a black hole with a clear horizon, leaving one blind to the location of microstates.

Thus, in order to try to understand the nature of the degrees of freedom one has to resort to other ideas, alternative to methods which try to find the entropy of a black hole from a fundamental theory. Following Bekenstein early idea [5], Bekenstein and Mukhanov [7] developed a heuristic scheme, where to each horizon Planck area one attaches a spin like degree of freedom, which provides a way to compute the number of accessible states, and thus the entropy, of a black hole.

This idea, that the degrees of freedom are on the horizon, has been put into a precise mathematical formulation with the works of Carlip [8, 9, 10, 11, 12, 13], which were developed and applied further by several authors, not only for general relativistic black holes [14, 15, 16, 17, 18, 19, 20], but also for Lovelock and other theories with black holes [21, 22, 23], as well as in cosmological models with horizons (see, e.g, [24]). Essentially, Carlip's suggestion is that the hair comes from the symmetries of the horizon. Now, the event horizon is not a true surface, and moreover one needs to know the global spacetime solution in order to trace its path. Therefore, the event horizon is not a candidate to extract information about the local micro degrees of freedom. On the other hand, the apparent horizon has a local meaning and thus can be used as the boundary surface where to impose boundary conditions and search for symmetries. The idea is that local quantum effects, manifested through Hawking radiation, break the diffeomorphism invariance of the spacetime and give sense to the prescribed boundary conditions in the vicinity of the apparent horizon. For instance, imposing the conditions that the canonical generators are differentiable at the boundary horizon gives rise to a 2-dimensional conformal field theory with a non-vanishing central charge $c$. This, together with the level zero conserved charge eigenvalue, $L_{0}$, of the conformal theory gives, through Cardy formula [25], which applies to 2-dimensional conformal field theories in general, the entropy of the black hole. One one hand, this idea has arisen from the problem of universality, which is to explain why all types of approaches 
mentioned above agree with the semiclassical computation [26]. A possible answer is the one advanced above, that the result for the black hole entropy is controlled by a symmetry on the horizon inherited from the classical theory, which turns out to be a conformal symmetry hiding many underlying specific degrees of freedom [8, 9, 10, 11, 12]. On the other hand, Carlip's method has its physical basis on an observation by Strominger [27], that by applying the Cardy formula [25] to the asymptotic symmetry group at infinity of the three-dimensional BTZ black hole spacetime [29], one recovers the Bekenstein-Hawking expression. This followed the early result of Brown and Henneaux [28], who showed that the asymptotic symmetry group at infinity of the three-dimensional anti-de Sitter spacetime is the conformal group in two dimensions with a non-vanishing central charge, and their additional remark that any quantum theory of such a type of spacetime should take this into account. The three-dimensional BTZ black hole [29] belongs to that class of spacetimes, i.e., 3-dimensional spacetimes asymptotically anti-de Sitter. However, this calculation is heavily dependent upon the $(2+1)$ dimensionality of the spacetime, (see [30] for an analysis along the same lines in $(1+1)$ dimensions), and it locates the states responsible for the entropy at infinity, giving only an upper bound for the entropy of anti-de Sitter spacetime in three dimensions (which, in this case, it is just as good, since the maximum of entropy in a region arises by inserting a black hole in it). The need to push these states onto the horizon resulted in the developments by Carlip [8, ㅇ, 10, 11, 12].

While putting the degrees of freedom on the horizon unequivocally, and in this way advancing that in order to understand the black hole entropy only the near horizon geometry of the black hole is relevant, Carlip's approach has not answered what those degrees of freedom are. Now, classically, black hole horizons are $(D-2)$-dimensional surfaces. Quantum mechanically they can fluctuate, so it is plausible to expect that these fluctuations can be described by effective field theories inhabiting the two dimensions transverse to the horizon, essentially the time and the radial dimensions. This idea has been put forward by Solodukhin [31], who suggested that a possible candidate for the horizon states, in spherical symmetric black holes, is indeed the set of degrees of freedom of spherically symmetric fluctuations of the $(D-2)$-dimensional horizon sphere. More precisely, the statistical origin of black hole entropy is in the set of states of a 2-dimensional conformal field theory describing the fluctuations of the $(D-2)$-dimensional surface. Thus, while Carlip suggests that the hair is in the symmetries of the horizon, Solodukhin realizes it on radial fluctuations of the 
horizon. Solodukhin reduced then the problem of $D$-dimensional black holes to an effective 2-dimensional theory with fixed boundary condition on the horizon. This theory, being conformal, admits then a chiral Virasoro algebra on the horizon, whose central charge $c$, and level zero operator eigenvalue $L_{0}$, yield directly, again through Cardy formula, the black hole entropy. This near horizon conformal field theory approach by Solodukhin [31] has been refined in [32], and extended to cosmological horizons like the de Sitter horizon [33], as well as to black hole horizons in the Lovelock theory [34]. Similar ideas have been developed in [35, 36].

Now, Solodukhin's method relies on some particular assumptions, one of them being spherical symmetry, and all the black holes studied up to now have spherical horizons. It is then worth understanding whether the topology of the horizon makes any difference to this method. We investigate whether it works for black holes whose horizons have topologies other than spherical, such as in electrically charged black holes in asymptotically locally antide Sitter spacetimes in which the $(D-2)$ horizon sphere (i.e, a compact and orientable $(D-2)$ surface of constant positive curvature) is replaced by a flat $(D-2)$ torus (i.e, a compact and orientable $(D-2)$ surface of zero curvature), or by a hyperbolic compact $(D-2)$ surface (i.e, a compact and orientable $(D-2)$ surface of constant negative curvature). In $D=4$ spacetime dimensions, the horizon can then be the usual 2-dimensional sphere (i.e., a Riemann surface with genus $q_{\text {enus }}=0$ ), or a 2-dimensional flat torus (i.e., a Riemann surface with genus $g_{\text {enus }}=1$ ) [37, 38|, or a 2-dimensional hyperbolic torus, (i.e, a Riemann surface with genus $g_{\text {enus }} \geq 2$ ) [39, 40, 41]. These 4-dimensional black hole solutions with compact orientable horizons have been generalized to $D$ dimensions in [42] (see [43] for further references). More specifically, in this article we widen Solodukhin's method to include a generic set of static, electrically charged black holes, with negative cosmological constant, and with horizons with spherical, flat-toroidal, and hyperbolic-compact surface topologies. This generic set of black holes with different topologies is introduced in Sec. II where the corresponding metrics, entropies, and temperatures are given. Note that, although the spherical case has been treated by Solodukhin [31], we include it here because the three topologies can be treated in a unified way. We then proceed in Sec. III to a dimensional reduction, performing first a $2+(D-2)$ splitting, and then integrating in the $(D-2)$ angular coordinates, obtaining finally an effective 2-dimensional theory on the horizon with the corresponding field equation and constraints. In Sec. IV] we show that there is conformal invariance on the horizon. Moreover, 
there exists an infinite number of conserved charges on the horizon, which provide a Virasoro algebra with non-vanishing central charge. This analysis applies to all dimensions and the three topologies. Using the Cardy formula we recover the Bekenstein-Hawking entropy. In Sec. $\nabla$ we state the conclusions and final remarks.

\section{THE BLACK HOLES}

\section{A. The action, and the metric and electric field}

The electrically charged anti-de Sitter black holes we are going to study are solutions of the Einstein-Maxwell action in $D$-dimensions, (we now put $\hbar=1, c=1$, and $k_{\mathrm{B}}=1$ ),

$$
I=\int d^{D} x \sqrt{-g} \mathcal{L}
$$

with $g$ being the determinant of the metric $g_{\mu \nu}$, and the Lagrangian $\mathcal{L}$ being composed of the gravitational part and the electromagnetic part, and given by

$$
\mathcal{L}=\frac{1}{16 \pi G}(R-2 \Lambda)-\frac{1}{4} F_{\alpha \beta} F^{\alpha \beta},
$$

where $G$ is the $D$-dimensional Newton constant, $R$ is the Ricci scalar constructed from the Riemann tensor $R_{\alpha \beta \gamma \delta}, \Lambda$ is the cosmological constant, $F_{\alpha \beta}$ is the Maxwell tensor, and greek indices run the entire spacetime coordinates $\alpha=0, \ldots, D-1$. Upon variation, the action (21)-(3) gives the Einstein equation

$$
R_{\alpha \beta}-\frac{1}{2} R g_{\alpha \beta}+\Lambda g_{\alpha \beta}=8 \pi G T_{\alpha \beta}
$$

where $R_{\alpha \beta}$ is the Ricci tensor and $T_{\alpha \beta}=\frac{1}{4 \pi}\left(F_{\alpha \gamma} F_{\beta}^{\gamma}-\frac{1}{4} g_{\alpha \beta} F_{\gamma \delta} F^{\gamma \delta}\right)$ is the electromagnetic energy-momentum tensor, and the Maxwell equation

$$
\nabla_{\alpha} F^{\alpha \beta}=0
$$

From Eq. (44) one can find a three-family of static, electrically charged black hole solutions with negative cosmological constant, with a parameter $k$, whose possible values are $k=$ $1,0,-1$. These three values correspond to black holes with spherical, flat-toroidal, and hyperbolic-toroidal topologies, respectively [37, 38, 39, 40, 41, 42, 43]. The horizons of these 
black holes are considered to be compact and orientable surfaces. The gravitational field of these black holes is described by the metric

$$
d s^{2}=-f(r) d t^{2}+f(r)^{-1} d r^{2}+r^{2}\left(d \Omega_{D-2}^{k}\right)^{2},
$$

where

$$
f(r)=k-\frac{\Lambda}{3} r^{2}-\frac{2 G M}{r^{D-3}}+\frac{G Q^{2}}{r^{2(D-3)}},
$$

with $D \geq 4$, and $M$ and $Q$ are proportional to the ADM mass and electric charge, respectively. For instance, in the spherical case and for zero cosmological constant one has $M=\frac{8 \pi m}{(D-2) \Sigma_{D-2}^{1}}$ and $Q^{2}=q^{2}\left(\frac{8 \pi}{(D-2)(D-3)}\right)$, where $m$ and $q$ are the ADM mass and electric charge, respectively, and $\Sigma_{D-2}^{1}$ is the area of the $(D-2)$ unit sphere [44]. For a generic dimension, and for generic types of black hole topologies, there is no closed expression for the ADM mass and electric charge, but for the matters we want to discuss here there is no need for such an expression. The angular part of (6) is given for each type of horizon, $k=1$, $k=0$, and $k=-1$, by

$$
\begin{aligned}
& \left(d \Omega_{D-2}{ }^{1}\right)^{2}=d \theta_{1}^{2}+\sin ^{2} \theta_{1} d \theta_{2}^{2}+\ldots+\prod_{i=1}^{D-3} \sin ^{2} \theta_{i} d \theta_{D-2}^{2}, \\
& \left(d \Omega_{D-2}^{0}\right)^{2}=d \theta_{1}^{2}+d \theta_{2}^{2}+\ldots+d \theta_{D-2}^{2}, \\
& \left(d \Omega_{D-2}^{-1}\right)^{2}=d \theta_{1}^{2}+\sinh ^{2} \theta_{1} d \theta_{2}^{2}+\ldots+\sinh ^{2} \theta_{1} \prod_{i=2}^{D-3} \sin ^{2} \theta_{i} d \theta_{D-2}^{2},
\end{aligned}
$$

respectively. In the spherical case, $k=1$, one can take the range of coordinates as the usual one $0 \leq \theta_{1}<\pi, 0 \leq \theta_{i}<2 \pi$ for $i \geq 2$. In the flat-torus case, $k=0$, the range is arbitrary, though finite for a compact surface, and we can choose $0 \leq \theta_{i}<2 \pi$. In the hyperbolic-compact surface case, $k=-1$, the situation is in general more involved. For instance, in $D=4$ spacetime dimensions, the horizon is a 2-dimensional hyperbolic torus, i.e, a Riemann surface with genus $g_{\text {enus }} \geq 2$. In $D=5$ spacetime dimensions, the horizon is a 3-dimensional compact manifold $\mathrm{M}^{3}$ of constant negative curvature. In this case, the 3-manifold $\mathrm{M}^{3}$ can be seen as a quotient space, $\mathrm{M}^{3}=\tilde{\mathrm{M}}^{3} / \Gamma$, where the universal covering $\tilde{\mathrm{M}}^{3}$ is the hyperbolic 3-surface, and $\Gamma$ is a discrete subgroup of the isometry group of $\mathrm{M}^{3}$ [42]. The range of coordinates in this case depends on $\Gamma$.

The electric field of these solutions is radial and is given by

$$
F_{a b}=F \epsilon_{a b}
$$


where $F$ is given by

$$
F=-\frac{1}{2} \sqrt{\frac{(D-3)(D-2)}{2 \pi}} \frac{Q}{r^{D-2}},
$$

and $\epsilon_{a b}$ is the antisymmetric tensor, with $a, b$ running through 0,1 , i.e., through the temporal $t$ and radial $r$ coordinates, respectively. We will split the greek indices $\alpha, \beta, \gamma, \ldots$ into two series of indices, the first denoted by lower case latin $a, b, c, \ldots=0,1$ for the temporal $t$ and radial $r$ coordinates, respectively, and the second by upper case latin $A, B, C, \ldots=$ $2, \ldots,(D-1)$ for the corresponding angular coordinates.

\section{B. The entropy}

The entropies associated with each topology all follow the area law. Indeed, according to [45, 46], one has for black holes with bifurcating horizons, such as those of (66)-(7), the following expression,

$$
S=-2 \pi \oint_{\Omega} d^{D-2} y \sqrt{-h} Y^{\alpha \beta \gamma \delta} \hat{\epsilon}_{\alpha \beta} \hat{\epsilon}_{\gamma \delta}
$$

with,

$$
Y^{\alpha \beta \gamma \delta} \equiv \frac{\partial \mathcal{L}}{\partial R_{\alpha \beta \gamma \delta}}
$$

$\Omega$ is the $(D-2)$-dimensional horizon surface spanned by the coordinates $y^{A}, A=2, \ldots,(D-$ 1). Here $d^{D-2} y \sqrt{-h}$ is the induced measure of integration, where $h$ is the determinant of the induced metric $h_{A B}$ calculated on the horizon, with $h_{A B}$ being the metric in the $(D-2)$ dimensional spacetime, such that $h_{A B} d y^{A} d y^{B}=r^{2}\left(d \Omega_{D-2}^{k}\right)^{2}$, and we have split the greek indices $\alpha, \beta, \gamma, \ldots$ into two series of indices, the first denoted by lower case latin $a, b, c, \ldots=0,1$ for the temporal $t$ and radial $r$ coordinates, respectively, and the second by upper case latin $A, B, C, \ldots=2, \ldots,(D-1)$ for the corresponding angular coordinates. $\mathcal{L}$ comes from Eq. (3). In addition, $R=R_{\alpha \beta \gamma \delta} g^{\alpha \gamma} g^{\beta \delta}=R_{\alpha \beta \gamma \delta} \frac{1}{2}\left(g^{\alpha \gamma} g^{\beta \delta}-g^{\beta \gamma} g^{\alpha \delta}\right)$, where the symmetries of the Riemann tensor $R_{\alpha \beta \gamma \delta}=R_{[\alpha \beta][\gamma \delta]}$ and $R_{\alpha \beta \gamma \delta}=R_{\gamma \delta \alpha \beta}$ were considered ([] denoting antisymetrization), and $\hat{\epsilon}_{\alpha \beta}$ is the binormal to the bifurcate Killing horizon, normalized as $\hat{\epsilon}_{\alpha \beta} \hat{\epsilon}^{\alpha \beta}=-2$. Now, following the definition (12), one has $Y^{\alpha \beta \gamma \delta}=\frac{1}{16 \pi G}\left(\frac{1}{2}\left(g^{\alpha \gamma} g^{\beta \delta}-g^{\beta \gamma} g^{\alpha \delta}\right)\right)$. After rearrangements we get $S=\frac{1}{4 G} \int_{\Omega} d^{D-2} y \sqrt{-h}$. Thus $\int_{\Omega} d^{D-2} y \sqrt{-h}=A_{D-2}$, where $A_{D-2}=\Sigma_{D-2}^{k} r_{\mathrm{h}}^{(D-2)}$ is the area of the $(D-2)$-dimensional horizon surface, $\Sigma_{D-2}^{k}$ being the area of the corresponding unit surface, with $k=1,0,-1$. The radius $r_{\mathrm{h}}$ is the horizon radius 
determined by the equality $f\left(r_{\mathrm{h}}\right)=0$. Thus,

$$
S=\frac{A_{D-2}}{4 G}
$$

in line with, e.g., the results in [40] found through other methods for four dimensions. The entropy depends only on the Einstein term of the action, which has the same form for all of these solutions. In four dimensions, $D=4$, and for spherical symmetry, (13) reduces to the Bekenstein-Hawking formula (11).

\section{The temperature}

Another important thermodynamic quantity for the black holes is the Hawking temperature $T$, the temperature at which the black holes emit black body radiation, which for these black holes is given by

$$
T=\frac{1}{4 \pi}\left[\frac{2(D-3) G M}{r_{\mathrm{h}}^{D-2}}-\frac{2(D-3) G Q^{2}}{r_{\mathrm{h}}^{2 D-5}}-\frac{2 \Lambda r_{\mathrm{h}}}{3}\right]
$$

This reduces to the Hawking temperature $T=1 /(8 \pi G M)$ for the 4-dimensional Schwarzschild black hole.

\section{DIMENSIONAL REDUCTION AND EFFECTIVE 2-DIMENSIONAL CON- FORMAL FIELD THEORY ON THE HORIZON}

\section{A. Preliminaries and motivation}

Now, suppose we are dealing with a spacetime containing a black hole, which still preserving the original spherical, toroidal, or hyperbolic symmetry, has a time dependence. This dependence could come from quantum vacuum fluctuations. Then a general class of $D$-dimensional metrics can be written as

$$
d s^{2}=d \sigma^{2}+d \Sigma^{2},
$$

where

$$
d \sigma^{2}=\gamma_{a b}(x) d x^{a} d x^{b},
$$


with $\gamma_{a b}(x)$ being a metric in a 2-dimensional spacetime with coordinates $x$, and where $x$ stands collectively for the time and spatial coordinates $x=\left(x^{0}, x^{1}\right)$, or, when convenient, for the null coordinates $x=\left(x^{+}, x^{-}\right)$, where $x^{+}=x^{0}+x^{1}$, and $x^{-}=x^{0}-x^{1}$, and

$$
d \Sigma^{2}=h_{A B}(x, y) d y^{A} d y^{B} \equiv r^{2}(x)\left(d \Omega_{D-2}^{k}\right)^{2},
$$

with $h_{A B}$ being the metric in the $(D-2)$-dimensional spacetime spanned by the coordinates $y$. The function $r(x)$ in Eq. (17) is the radial function, a type of dilaton function, in general dependent on the coordinates $x$. Note that the metrics (66)-(86), being time independent, are a special case of this class of metrics. Indeed, the 2-dimensional part of the metrics (6) - (8) can be represented by the 2-dimensional metric $d \sigma^{2}=\gamma_{a b}(x) d x^{a} d x^{b}$, appearing in Eqs. (15)-(16), as

$$
d \sigma^{2}=-f\left(x^{1}\right)\left(d x^{0}\right)^{2}+\frac{\left(d x^{1}\right)^{2}}{f\left(x^{1}\right)},
$$

with the function $f\left(x^{1}\right)$ vanishing at $x^{1}=x_{\mathrm{h}}^{1}$, where $x_{\mathrm{h}}^{1}$ is the horizon coordinate. In lightcone coordinates, one has $d \sigma^{2}=f\left(x_{+}, x_{-}\right) d x^{+} d x^{-}$. For non-extremal black holes, one can write the function $f$ near the horizon as,

$$
f\left(x^{1}\right)=\frac{4 \pi}{\beta_{\mathrm{h}}}\left(x^{1}-x_{\mathrm{h}}^{1}\right)+O\left(\left(x^{1}-x_{\mathrm{h}}^{1}\right)^{2}\right),
$$

where $\beta_{\mathrm{h}}=1 /\left(k_{\mathrm{B}} T\right)$ is the inverse temperature parameter, with $T$ given in Eq. (14).

Now, the event horizon of a black hole is not a true surface, but the apparent horizon $\mathcal{H}$ has a local meaning and thus can be used as the boundary surface where to impose asymptotic boundary conditions. The apparent horizon $\mathcal{H}$ for metrics (15)-(17) is defined as the surface $r(x)=$ constant whose outward normals are null, i.e.,

$$
\left.\gamma^{a b}\left(\nabla_{a} r\right)\left(\nabla_{b} r\right)\right|_{\mathcal{H}}=0,
$$

where $\nabla$ is the covariant derivative with respect to $\gamma_{a b}$. This is a condition on the dilaton function $r(x)$. Note that condition (20) does not change under conformal transformations of the 2-dimensional metric $\gamma_{a b}$, i.e., transformations of the type $\gamma_{a b} \rightarrow \mathrm{e}^{2 \rho} \gamma_{a b}$. This led Solodukhin [31] to consider condition (20) as saying that the dynamics of the fluctuating metric is solely in the radial-dilaton function $r(x)$, while $\gamma_{a b}$ represents a class of conformal, but otherwise fixed, metrics. It was further shown by Carlip [32] that the condition " $\gamma_{a b}$ non-dynamical" is consistent throughout. Interesting enough, Giacomini and Pinamonti 
[35, 36] have imposed otherwise, that $\gamma_{a b}$ is dynamical and the radial-dilaton function is non-dynamical, having found an effective, non-Lagrangian, theory which can be used to extract the horizon properties.

Further arguments, which also apply to the spacetimes we are studying, led Solodukhin [31] to conclude that the generators $l_{\xi} \equiv \xi^{a} \partial_{a}$ of diffeomorphisms which preserve condition (20), and thus preserve the horizon $\mathcal{H}$, are conformal Killing vectors, satisfying the conformal

Killing equation $\nabla_{a} \xi_{b}+\nabla_{b} \xi_{a}=\frac{1}{2} \gamma_{a b} \nabla_{c} \xi^{c}$, generating thus the infinite dimensional group of conformal transformations on the horizon. Now, in general, the horizon $\mathcal{H}$ has several components, for instance, for the static black holes mentioned in Sec. II the horizon is composed of a future component $\mathcal{H}_{\mathrm{f}}$, defined in null coordinates by $x_{-}=0$ and $x_{+}>0$, and a past component $\mathcal{H}_{\mathrm{p}}$, defined by $x_{+}=0$ and $x_{-}<0$. In the static case these components intersect. We will be interested in the future horizon $\mathcal{H}_{\mathrm{f}}$. In this case, the future generators of $\mathcal{H}_{\mathrm{f}}$ are given by $l_{\xi}=\xi^{+}\left(x^{+}\right) \partial_{+}$. In turn, its Fourier components $l_{n}=\mathrm{e}^{i n x^{+}} \partial_{+}$form a copy of the Virasoro algebra, i.e., of $\left[l_{n}, l_{m}\right]=i(m-n) l_{n+m}$, with respect to the Lie bracket $\left[\xi_{1}, \xi_{2}\right]=\left(\xi_{1} \xi_{2}^{\prime}-\xi_{2} \xi_{1}^{\prime}\right)[31]$.

These arguments show unequivocally that the horizon has some conformal structure built in it, and leads one to seek an effective field theory near the horizon of a black hole. Solodukhin [31] worked out in detail the case of spherically symmetric general relativity and showed that treating the horizon as a boundary leads to a theory, that is indeed a conformal theory possessing the Virasoro algebra of the generators of the horizon, plus a central extension, with a central charge which is a crucial term for finding the entropy of the black hole. We will work out in detail black holes with toroidal and hyperbolic topology in general relativity (including for completeness the spherical case first treated by Solodukhin [31]) and show that all the different topologies yield a conformal field theory at the horizon whose extended Virasoro algebra possesses also a central term.

\section{B. Dimensional reduction and effective 2-dimensional conformal theory}

In order to find an effective 2-dimensional field theory, we now apply dimensional reduction to the action (2) using the $2+(D-2)$ splitting used in Eq. (15) for the metrics (6) -(8) . Since we have agreed that the dynamical field is the radial coordinate $r$, this will be kept in the action. Then, one first dimensionally reduces the gravitational part of the action (2) 
to a 2-dimensional spacetime represented by (16). In addition, one also has to reduce the electromagnetic part of the action. Dimensionally reducing the action (2) gives then

$$
\begin{aligned}
I=-\frac{\sum_{D-2}^{k}}{16 \pi G} \int d^{2} x \quad & \sqrt{-\gamma}\left[r^{D-2} R+(D-3)(D-2) r^{D-4}(\nabla r)^{2}+\right. \\
& \left.k(D-3)(D-2) r^{D-4}-2 \Lambda r^{D-2}+8 \pi G F^{2} r^{D-2}\right],
\end{aligned}
$$

where $R$ is now the scalar curvature in two dimensions, $\gamma_{a b}$ is the 2-dimensional metric, $\Lambda$ is still the cosmological constant, $\Sigma_{D-2}^{k}$ is the area of the corresponding unitary surface, a factor that comes from the integration of the angular coordinates, and $F$ is given in Equation (10). For the spherical case, $k=1$, one gets $\Sigma_{D-2}^{1}=2 \pi^{\frac{D-1}{2}} / \Gamma\left(\frac{D-1}{2}\right)$. For the flat-torus case, $k=0$, choosing the range of angular coordinates between 0 and $2 \pi$, gives $\Sigma_{D-2}^{0}=(2 \pi)^{D-2}$. For the hyperbolic-compact surface case, $k=-1$, the situation is more complicated. For a 2-dimensional compact hyperbolic horizon (in the spacetime with $D=4$ ), one has, by the Gauss-Bonnet theorem, $\Sigma_{2}^{-1}=4 \pi\left(g_{\text {enus }}-1\right)$, where $g_{\text {enus }}$ is the genus of the Riemann surface, with $g_{\text {enus }} \geq 2$. For higher horizon dimensions, the area of the unitary compact surface depends on the isometry group one considers acting on the hyperbolic space [42].

Now redefine the coordinate $r$ as the following dilaton field $\phi$,

$$
\phi=\frac{1}{2 q}\left(\frac{D-2}{D-3}\right) C^{\frac{1}{2}}\left(\frac{r^{2}}{r_{\mathrm{h}}}\right)^{\frac{D-2}{2}}
$$

where $q$ is a constant to be determined later, and $C=\frac{\Sigma_{D-2}^{k}}{2 \pi G}\left(\frac{D-3}{D-2}\right)$, and perform a conformal transformation of the 2-dimensional metric $\gamma_{a b}$, into a metric $\bar{\gamma}_{a b}$,

$$
\gamma_{a b}=\left(\frac{\phi_{\mathrm{h}}}{\phi}\right)^{\frac{D-3}{D-2}} \mathrm{e}^{\frac{1}{q^{2}\left(\frac{D-3}{D-2}\right)} \frac{\phi}{\phi_{\mathrm{h}}}} \bar{\gamma}_{a b}
$$

where $\phi_{\mathrm{h}}=\frac{1}{2 q} C^{\frac{1}{2}}\left(\frac{D-2}{D-3}\right)\left(r_{\mathrm{h}}\right)^{\frac{D-2}{2}}$ is the classical value of the field on the horizon. Then the action (21) becomes a Liouville type action,

$$
I=-\int d^{2} x \sqrt{-\bar{\gamma}}\left[\frac{1}{2}(\bar{\nabla} \phi)^{2}+\frac{1}{2} q^{2}\left(\frac{D-3}{D-2}\right) \phi_{\mathrm{h}} \phi \bar{R}+U(\phi)\right],
$$

where $\bar{\nabla}$ is the derivative associated with the new metric $\bar{\gamma}_{a b}, \bar{R}$ is the scalar curvature associated with $\bar{\gamma}$, derived from the original scalar $R$ through $\bar{R}=\Omega^{-2}\left[R-2(D-1) \gamma^{a b} \nabla_{a} \nabla_{b}(\ln \Omega)-(D-2)(D-1) \gamma^{a b}\left(\nabla_{a} \ln \Omega\right)\left(\nabla_{b} \ln \Omega\right)\right]$, with $\Omega=$ 


$$
\begin{aligned}
\left(\frac{\phi}{\phi_{\mathrm{h}}}\right)^{\frac{D-3}{2(D-2)}} \exp \left(-\frac{\phi}{2 q^{2}\left(\frac{D-3}{D-2}\right) \phi_{\mathrm{h}}}\right)(\mathrm{cf.}, \text { e. g., }[3]) \text {, and where the potential } U(\phi) \text { is given by } \\
\begin{aligned}
U(\phi)=\left(\frac{\phi_{h}}{\phi}\right)^{\left(\frac{D-3}{D-2}\right)} e^{\frac{\phi}{q^{2}\left(\frac{D-3}{D-2}\right) \phi_{\mathrm{h}}}} & {\left[\frac{k}{2}(D-2)^{2} C^{\frac{2}{D-2}}\left(\frac{D-3}{D-2}\right)^{2} q^{2} \phi_{\mathrm{h}} \phi\right.} \\
& \left.+q^{2}\left(\frac{D-3}{D-2}\right) \phi_{\mathrm{h}} \phi\left(4 \pi G F^{2}-\Lambda\right)\right]
\end{aligned}
\end{aligned}
$$

Except for the metric $\bar{\gamma}_{a b}$, in the subsequent expressions the bar in the several other quantities will be dropped for simplicity of notation.

The action (24) provides an effective theory for the dilaton field $\phi$, which in turn represents the oscillations of the radial coordinate near the horizon. To find the equations of these oscillation one has to vary the action (24). Its variation with respect to the dilaton field gives the following equation of motion for $\phi$ itself,

$$
\square \phi=\frac{1}{2} q^{2}\left(\frac{D-3}{D-2}\right) \phi_{\mathrm{h}} R+U^{\prime}(\phi),
$$

where $\square$ is the d'Alembertian $\square=\partial_{a} \partial^{a}$. The variation of the action (24) with respect to the metric $\bar{\gamma}_{a b}$ gives $\delta I=\frac{1}{2} \int d^{2} x T^{a b} \delta \bar{\gamma}_{a b}=0$, i.e.,

$$
\begin{aligned}
T_{a b} \equiv & \frac{1}{2} \partial_{a} \phi \partial_{b} \phi-\frac{1}{4} \bar{\gamma}_{a b}(\nabla \phi)^{2}+\frac{1}{2} q^{2}\left(\frac{D-3}{D-2}\right) \phi_{\mathrm{h}}\left(\bar{\gamma}_{a b} \square \phi-\nabla_{a} \nabla_{b} \phi\right) \\
& -\frac{1}{2} \bar{\gamma}_{a b} U(\phi)=0
\end{aligned}
$$

where we have used that the Einstein tensor $G_{a b}=R_{a b}-\frac{1}{2} \bar{\gamma}_{a b} R$ is identically zero in two dimensions. This equation is a constraint equation.

So, now one has a 2-dimensional theory, with constraints, for a scalar field $\phi$, strikingly resembling bosonic string theory [47]. However, there is a snag; as it is, this theory is not conformally invariant everywhere. Indeed, the trace of $T_{a b}, T_{a}^{a}=\bar{\gamma}^{a b} T_{a b}$, is given by

$$
T_{a}^{a}=\frac{1}{2} q^{2}\left(\frac{D-3}{D-2}\right) \phi_{\mathrm{h}} \square \phi-U(\phi)
$$

and is not equal to zero in general. Now, under conformal transformations, $\bar{\gamma}_{a b} \rightarrow \bar{\gamma}_{a b}(x)+$ $\lambda(x) \bar{\gamma}_{a b}(x)$, one has $\delta \bar{\gamma}_{a b}=\lambda(x) \bar{\gamma}_{a b}(x)$, and so the action varies as $\delta I=\frac{1}{2} \int d^{2} x \lambda(x) T_{a}^{a}$. Thus, one sees that the vanishing of the trace of the energy-momentum tensor is a necessary condition for invariance of the action under conformal transformations. This means that the 2-dimensional theory for the scalar $\phi$ is not, in general, a conformal theory. Interestingly enough, it becomes conformal in an infinitesimally small vicinity of the horizon. 
To see this, choose, close to the horizon, the following coordinate $z$

$$
z=\int^{x^{1}} \frac{d x}{f(x)}=\frac{\beta_{\mathrm{h}}}{4 \pi} \ln \left(x^{1}-x_{\mathrm{h}}^{1}\right),
$$

where $f(x)$ is given by Eq. (19). Note that this asymptotic region, close to the horizon, is a region of negative high values of $z, z \rightarrow-\infty$. The metric function $f(x)$ given in Eq. (19) becomes,

$$
f(z)=f_{0} e^{\frac{4 \pi}{\beta_{\mathrm{h}}} z},
$$

where $f_{0}$ is some constant. Thus $f(z)$ vanishes exponentially fast at the horizon. We can see that after this transformation the metric has gone from $\bar{\gamma}_{a b}$ to a metric of the form $f(z) \eta_{a b}$, where $\eta_{a b}$ is the Minkowski metric in two dimensions. We also put $x^{0}=t$, so that the 2dimensional plane is now defined by the coordinates $(t, z)$. Then, Eq. (26) is, in coordinates $(t, z)$, given by

$$
-\partial_{t}^{2} \phi+\partial_{z}^{2} \phi=\frac{1}{2}\left(\frac{D-3}{D-2}\right) q^{2} \phi_{\mathrm{h}} R f(z)+f(z) U^{\prime}(\phi) .
$$

At the horizon, for large negative values of $z$, the right hand side of Eq. (31) vanishes exponentially fast. It then becomes, at $z \rightarrow-\infty$,

$$
\partial_{t}^{2} \phi-\partial_{z}^{2} \phi=0
$$

a simple d'Alembert wave equation. Of course, this equation is conformally invariant, since if we choose null coordinates $z^{+}=t+z$ and $z^{-}=t-z$ one obtains

$$
\partial_{-} \partial_{+} \phi=0
$$

and the change to coordinates ${z^{+}}^{\prime}=f\left(z^{+}\right)$and $z^{-\prime}=f\left(z^{-}\right)$, which is a conformal transformation in two dimensions, yields the same wave equation (33) for the scalar field. Moreover, using Eq. (30) when $z \rightarrow-\infty$, and Eq. (32), one finds that $T_{+-}=T_{-+}=0$, and thus the trace of $T_{a b}$ in coordinates $\left(z^{+}, z^{-}\right)$, for large negative $z$, now obeys

$$
T_{-+}+T_{+-}=0
$$

i.e., it vanishes on-shell, confirming that the field $\phi$ is now described by a conformal field theory in an arbitrary small vicinity of the horizon [31]. Note that the conformal field theory is classical, since the trace $T_{a}^{a}$, given in (34), vanishes only when the equations of motion 
for $\phi$ are obeyed. Note also that on the horizon the field $\phi$ is only a function, either of the coordinate $z^{+}$, or of $z^{-}$. Since we are interested in the future branch of the horizon $\mathcal{H}_{\mathrm{f}}$ (see Sec. IIIA), we take $\phi$ to be a function of $z^{+}$. This will make the conformal field theory a chiral theory.

\section{CONFORMAL ENTROPY}

We are now ready to find the entropy, via conformal means, of these three classes of black holes, spherical, toroidal, and hyperbolic black holes.

The conservation law for the energy-momentum tensor, $\partial_{a} T^{a b}=0$, in null coordinates is $\partial_{-} T_{++}+\partial_{+} T_{-+}=0$. As $T_{-+}=0$, this conservation law reads

$$
\partial_{-} T_{++}=0
$$

Interchanging $-\leftrightarrow+$ one also finds, $\partial_{+} T_{--}=0$. Classically, incoming motion from the horizon is forbidden, so one should use only the component $T_{++}$of the stress tensor as the physical charge. The corresponding charge generates the conformal transformations. That is, if one has a function of $z^{+}$, e.g., $g\left(z^{+}\right)$, Eq. (35) implies that $g T_{++}$is conserved, $\partial_{-}\left(g T_{++}\right)=0$. Then, the charge $Q_{g}=\int d z g\left(z^{+}\right) T_{++}$is conserved, and there is an infinite set of them as well, for there is an infinite number of functions $g$. This happens only in two dimensions. The resemblance with bosonic string theory is now complete.

The function $g\left(z^{+}\right)$can be identified with $\xi^{+}\left(z^{+}\right), g\left(z^{+}\right) \equiv \xi^{+}\left(z^{+}\right)$, where $\xi^{+}\left(z^{+}\right)$is the non-zero component of the generators of the conformal transformation $l_{\xi}=\xi^{+} \partial_{+}$which preserve the horizon condition (20), (see subsection (IIIA) ). Now, since we are interested in conserved quantities, these have the same value for any $t$, so we can calculate them at $t=0$, where the generators are only functions of $z$, i.e, $\xi^{+} \partial_{+}=\xi(z) \partial_{z}$, where we have also simplified the notation putting $\xi^{+}=\xi$. Thus the conserved charge associated with the infinitesimal conformal generators on the horizon $\xi \partial_{z}$ is then

$$
Q_{\xi}=\int \xi(z) T_{++}
$$

where $T_{++}$is evaluated at an infinitesimal vicinity of the horizon, also at $t=0$, such that $T_{++}=T_{++}(z)$, and can be found from Eq. (27), near the horizon. Since the Poisson algebra

of theses charges, $\left\{Q_{\xi_{1}}, Q_{\xi_{2}}\right\}$, is an expression of fundamental properties of the corresponding 
field theory, in this case a conformal one, one hopes that from this one obtains fundamental properties of the horizon. In order to obtain this algebra, note that from the action (24) one finds that the momentum $\pi$ conjugate to the field $\phi$ is $\pi=\delta \mathcal{L} / \delta\left(\partial_{t} \phi\right)=\partial_{t} \phi$, where $\mathcal{L}$ is the Lagrangian associated to the action. Since the Poisson algebra for the two fundamental fields $\phi$ and $\pi$ is defined as

$$
\begin{aligned}
\left\{\phi(z, t), \pi\left(z^{\prime}, t\right)\right\} & =\int d y\left(\frac{\delta \phi(z, t)}{\delta \phi(y, t)} \frac{\delta \partial_{t} \phi\left(z^{\prime}, t\right)}{\delta \partial_{t} \phi(y, t)}-\frac{\delta \phi(z, t)}{\delta \partial_{t} \phi(y, t)} \frac{\delta \partial_{t} \phi\left(z^{\prime}, t\right)}{\delta \phi(y, t)}\right) \\
& =\delta\left(z-z^{\prime}\right),
\end{aligned}
$$

one finds,

$$
\left\{Q_{\xi_{1}}, Q_{\xi_{2}}\right\}=Q_{\left[\xi_{1}, \xi_{2}\right]}+\left(\frac{q^{2} \phi_{\mathrm{h}}}{4}\right)^{2} \int C\left[\xi_{1}, \xi_{2}\right] d z,
$$

where $\left[\xi_{1}, \xi_{2}\right]=\xi_{1} \xi_{2}^{\prime}-\xi_{2} \xi_{1}^{\prime}$, as before, with ${ }^{\prime} \equiv \partial_{z}$, and $C\left[\xi_{1}, \xi_{2}\right] \equiv\left(\xi_{1}^{\prime}+\beta_{\mathrm{h}}^{-1} \xi_{1}\right)\left(\xi_{1}^{\prime}+\beta_{\mathrm{h}}^{-1} \xi_{2}\right)^{\prime}-$ $\left(\xi_{1}^{\prime}+\beta_{\mathrm{h}}^{-1} \xi_{1}\right)^{\prime}\left(\xi_{1}^{\prime}+\beta_{\mathrm{h}}^{-1} \xi_{2}\right)$. This is algebra is valid only in a close vicinity the horizon.

Note that within the context of the pure conformal field theory (32), once it is granted that the field $\phi$ obeys a conformal field theory, the coordinate $z$ has a range $-\infty \leq z \leq+\infty$. In order to proceed, and be able to use standard techniques, one imposes periodic boundary conditions on the field, i.e., the field is confined within a box of length $L$, say, with an infinite number of boxes joined smoothly together so that the range of $z$ is still infinite. One can then concentrate in one box only of length $L$. The length $L$ must have a physical meaning. In the context we are working with, the only quantum length scale that appears in the problem is the inverse Hawking temperature $\beta_{\mathrm{h}}$, which can be interpreted as the associated Compton thermal wavelength. As we will see this identification will appear naturally within this formalism. The best way to realize periodic boundary conditions here it is to compactify the coordinate $z$ on a circle of circumference $L$, such that $z$ lies in the interval $-\frac{L}{2} \leq z \leq \frac{L}{2}$.

Then, the functions $\xi(z)$ can be expanded in Fourier modes as $\xi(z)=\sum a_{n} e^{i \frac{2 \pi}{L} n z}$, where the $a_{n}$ are complex numbers. So, a basis for $\xi$ can be taken as $\xi_{n}=e^{i \frac{2 \pi}{L} n z}$, i.e., we are looking at diffeomorphisms with period $L$. Then, the conserved charges $Q_{\xi}$ turn into conserved charges $Q_{n}$, given by

$$
Q_{n}=\frac{L}{2 \pi} \int_{-L / 2}^{L / 2} d z e^{i \frac{2 \pi}{L} n z} T_{++} .
$$

These charges generate the following Poisson algebra

$$
i\left\{Q_{k}, Q_{n}\right\}=(k-n) Q_{n+k}+\frac{c}{12}\left[k^{3}+\left(\frac{L}{2 \pi \beta_{\mathrm{h}}}\right)^{2} k\right] \delta_{n+k, 0},
$$


which is a Virasoro type algebra, with

$$
c=12 \pi q^{4}\left(\frac{D-3}{D-2}\right)^{2} \phi_{\mathrm{h}}^{2},
$$

being the central charge.

In order to transform (40) into the usual form of the Virasoro algebra one performs a shift from the generators $Q_{n}$ to generators $L_{n}$, given by

$$
L_{n}=Q_{n}+\frac{c}{24}\left[\left(\frac{L}{2 \pi \beta_{\mathrm{h}}}\right)^{2}+1\right] \delta_{n, 0} .
$$

The Virasoro generators $L_{n}$, which obey an algebra with a central charge, are prompt to be quantized. Note first that, near the horizon, all the functions depend only on the coordinate $z^{+}=t+z$, so that the periodicity properties in time $t$ should be identical to those in $z$. Then, since the most natural scale in the problem is the imaginary time period of the Euclidean black hole, one should set the length scale $L$ equal to it, $L=2 \pi \beta_{\mathrm{h}}$. Thus, Eq. (42) turns into

$$
L_{n}=Q_{n}+\frac{c}{12} \delta_{n, 0}
$$

Second, to quantize the generators, one swaps the Poisson brackets for quantum commutators, through $[]=,i \hbar\{$,$\} , and divides the generators by \hbar$. Then, with the help of Eq. (40) one finds that the conserved charge operators $L_{n}$, usually called Virasoro operators, satisfy the usual Virasoro algebra,

$$
\left[L_{k}, L_{n}\right]=(k-n) L_{n+k}+\frac{c}{12}\left(k^{3}-k\right) \delta_{k+n, 0} .
$$

Note that for $\phi_{\mathrm{h}}=0$, the case with no black hole, and thus no central charge, $c=0$, this set up gives the algebra of the conformal group on a classical massless scalar field $\phi$ in two dimensions, which is known to be a conformally invariant theory.

Given that one has a Virasoro algebra for the conserved charge operators, one can now use the Cardy formula, which yields the number of asymptotic states of a conformal field theory. Its logarithm is the associated entropy, given by [25],

$$
S_{\mathrm{conf}}=2 \pi \sqrt{\frac{c}{6}\left(L_{0}-\frac{c}{24}\right)},
$$

where $L_{0} \equiv L_{\left.n\right|_{n=0}}$ is now considered as the eigenvalue of the corresponding Virasoro operator. 
To find $L_{0}$ we first find $Q_{0}$ through Eq. (39). Near the horizon $\phi \approx \phi_{\mathrm{h}}$, so in the semi-classical approximation, one has from (27) that $T_{++}$is given by

$$
\begin{aligned}
T_{++} & =\frac{1}{4}\left(\partial_{t} \phi+\partial_{z} \phi\right)^{2} \\
& -\frac{1}{2}\left(\frac{D-3}{D-2}\right) q^{2} \phi_{\mathrm{h}}\left[\partial_{z}\left(\partial_{t} \phi+\partial_{z} \phi\right)-\frac{2 \pi}{\beta_{\mathrm{h}}}\left(\partial_{t} \phi+\partial_{z} \phi\right)\right] .
\end{aligned}
$$

Then integration of $T_{++}$along the coordinate $z$, compactified in a circle of circumference $L$, gives zero. Since $Q_{0}=\frac{L}{2 \pi} \int_{-L / 2}^{L / 2} d z T_{++}$, one has $Q_{0}=0$. This is a consequence of the periodicity of the coordinate $z$ and of the fact that the integration is to be performed in the interval $-L / 2<z<L / 2$. Indeed, first note that $\int_{-L / 2}^{L / 2} d z \partial_{z} \phi=\left.\phi\right|_{-L / 2} ^{L / 2}=0$, by periodicity. One also finds $\int_{-L / 2}^{L / 2} d z \partial_{t} \phi=\partial_{t} \int_{-L / 2}^{L / 2} d z \phi=0$ by periodicity. The same applies to the case where there are second derivatives, or squares of first derivatives. For the case where we have mixed products, $\partial_{t} \phi \partial_{z} \phi$, we can see that $\partial_{t} \phi \partial_{z} \phi=\partial_{t}\left(\phi \partial_{z} \phi\right)-\phi \partial_{t} \partial_{z} \phi$. Using $\int_{-L / 2}^{L / 2} d z \phi \partial_{z} \phi=\left.\frac{1}{2} \phi^{2}\right|_{-L / 2} ^{L / 2}=0$ and $\int_{-L / 2}^{L / 2} d z \partial_{z} \phi \partial_{t} \phi=\left.\frac{1}{4} \partial_{t}\left(\phi^{2}\right)\right|_{-L / 2} ^{L / 2}=0$ we conclude that the charge $Q_{0}=0$. Then from Eq. (43) one finds that

$$
L_{0}=\frac{c}{12}
$$

Thus Solodukhin's method allows to obtain a relation between the eigenvalue $L_{0}$ and the central charge $c$, but not their values independently. Since the central charge depends on the unknown conformal parameter $q$, defined in Eqs. (22)-(23), the conformal entropy given by the Cardy formula (45) is determined up to the parameter $q$. Indeed, one has

$$
S_{\mathrm{conf}}=2 \pi^{2} q^{4}\left(\frac{D-3}{D-2}\right)^{2} \phi_{\mathrm{h}}^{2}
$$

Comparing the gravitational entropy given in the Eq. (13), written in the form

$$
S=\frac{A_{D-2}}{4 G}=\frac{\Sigma_{D-2}^{, k} r_{\mathrm{h}}^{D-2}}{4 G}=2 \pi q^{2}\left(\frac{D-3}{D-2}\right) \phi_{\mathrm{h}}^{2},
$$

with the conformal entropy (48), gives that

$$
S_{\mathrm{conf}}=\left[q^{2}\left(\frac{D-3}{D-2}\right) \pi\right] S .
$$

The parameter $q$ in Eqs. (22)-(23) is a free parameter in the theory. It is a trade between fluctuations in the field $\phi$ and distance measurements through the metric $\overline{\gamma_{a b}}$. For small $q$ the fluctuations are large and the measurements small, yielding a large horizon entropy; for 
large $q$ the fluctuations are small and the measurements large, yielding a small entropy. If one chooses

$$
q^{2}=\left(\frac{D-2}{D-3}\right) \frac{1}{\pi},
$$

one obtains precisely the Bekenstein-Hawking entropy.

\section{CONCLUSIONS AND REMARKS}

In this analysis we have shown that the method of Solodukhin [31] may be applied to the more complete set of static black holes within general relativity, namely, black holes not only with a spherical horizon, but also with toroidal and hyperbolic compact horizons. We have shown that these additions, i.e., the introduction of different topologies for the horizon, were absorbed by the effective potential into the equations, which eventually disappear in the infinitesimal vicinity of the horizon. Thus, one obtains for the conformal entropy of the black hole that the Bekenstein-Hawking formula, i.e., the entropy is proportional to the horizon area, holds for any of the topologies considered within this formalism. This is another instance where the universality of the black hole entropy concept, as formulated in [26], applies.

On a more generic vein, one can remark that the horizon, through the boundary conditions imposed on it, gives a conformal character to the oscillatory dilaton field in its vicinity. This is no surprise, since it is known that, in general, the fields become massless asymptotically close to the horizon (see, e.g, [48] for another instance of a scalar field propagation turning into a conformal field theory in the vicinity of the horizon). What, perhaps, is surprising is that the corresponding classical conformal theory has a central charge, which upon quantization, and through appropriate methods gives, through Cardy formula, the black hole entropy as found in $[13,31,33,34]$ and here. It is also worth noting that the Cardy formula applies to any conformal field theory in two dimensions, be it derived from condensed matter physics, quantum field theory, or gravity theories. Explaining why very different theories, that nonetheless yield the same central charge and the same level operator $L_{0}$, have, through the Cardy formula, the same entropy, is no easy task, up to now there being no clear physical argument for that. This is perhaps a consequence of the fact that the Cardy formula itself is hard to interpret [49]. an exception being the scalar field where a clear physical interpretation can be provided [50]. 


\section{Acknowledgments}

This work was partially funded by Fundação para a Ciência e a Tecnologia (FCT) of the Ministry of Science, Portugal, through project POCTI/FIS/57552/2004. GASD is supported by grant SFRH/BD/2003 from FCT. JPSL thanks Observatório Nacional do Rio de Janeiro for hospitality. 
[1] J. Bekenstein, Phys. Rev. D 7, 2333 (1973).

[2] S. Hawking, Comm. Math. Phys. 43, 199 (1975).

[3] R. M. Wald, General Relativity, (University of Chicago Press, Chicago, 1984).

[4] J. P. S. Lemos, gr-qc/0507101

[5] J. Bekenstein, Lett. Nuov. Cimento 11, 467 (1974).

[6] A. Strominger and C. Vafa, Phys. Lett. B 379, 99 (1996).

[7] J. D. Bekenstein and V. F. Mukhanov, Phys. Lett. B 360, 7 (1995).

[8] S. Carlip, Phys. Rev. Lett. 82, 2828 (1999).

[9] S. Carlip, Classical Quantum Gravity 16, 3327 (1999).

[10] S. Carlip, Classical Quantum Gravity 17, 4175 (2000).

[11] S. Carlip, Phys. Rev. Lett. 88, 241301 (2002).

[12] S. Carlip, Classical Quantum Gravity 22, 1303 (2005).

[13] S. Carlip, gr-qc/0508071

[14] M.-I. Park and J. Ho, Phys. Rev. Lett. 83, 5595 (1999).

[15] V. O. Soloviev, Phys. Rev. D 61, 027502 (1999).

[16] M.-I. Park and J. H. Yee, Phys. Rev. D 61, 088501 (2000).

[17] O. Dreyer, A. Ghosh, and J. Wisniewski, Classical Quantum Gravity 18, 1929 (2001).

[18] J. Koga, Phys. Rev. D 64, 124012 (2001).

[19] M.-I. Park, Nucl. Phys. B 634, 339 (2002).

[20] G. Kang, J Koga, and M.-I. Park, Phys. Rev. D 70, 024005 (2004).

[21] M. Cvitan, S. Pallua, and P. Prester, Phys. Lett. B 555, 248 (2003).

[22] M. Cvitan, S. Pallua, and P. Prester, Phys. Lett. B 571, 217 (2003).

[23] M. Cvitan and S. Pallua, Phys. Rev. D 71, 104032 (2005).

[24] R. Brustein, Phys. Rev. Lett. 86, 576 (2001).

[25] J. Cardy, Nucl. Phys. B 270, 186 (1986).

[26] S. Carlip, Nucl. Phys. Proc. Suppl. 57, 12 (1997).

[27] A. Strominger, JHEP 9802, 009 (1998).

[28] J. D. Brown and M. Henneaux, Commun. Math. Phys. 104, 207 (1985).

[29] M. Bañados, C. Teitelboim, and J. Zanelli, Phys. Rev. Lett. 69, 1849 (1992). 
[30] M. Cadoni and S. Mignemi, Phys. Rev. D 59, 081501 (1999).

[31] S. N. Solodukhin, Phys. Lett. B 454, 213 (1999).

[32] S. Carlip, Phys. Lett. B 508, 168 (2001).

[33] F.-L. Lin and Y.-S. Wu, Phys. Lett. B 453, 222 (1999).

[34] M. Cvitan, S. Pallua, and P. Prester, Phys. Lett. B 546, 119 (2002).

[35] A. Giacomini and N. Pinamonti, JHEP 0302, 014 (2003).

[36] A. Giacomini, hep-th/0405120.

[37] J. P. S. Lemos, Phys. Lett. B textbf353, 46 (1995).

[38] J. P. S. Lemos and V. T. Zanchin, Phys. Rev. D 54, 3840 (1996).

[39] W. L. Smith and R. B. Mann, Phys. Rev. D 56, 4942 (1997).

[40] D. R. Brill, J. Louko, and P. Peldán, Phys. Rev. D 56, 6475 (1997).

[41] L. Vanzo, Phys. Rev. D 56, 3600 (1997).

[42] D. Birmingham, Class. Quant. Grav. 16, 1197 (1999).

[43] N. L. Santos, O. J. C. Dias, and J. P. S. Lemos, Phys. Rev. D 70, 124033 (2004).

[44] R. C. Myers and M. J. Perry, Ann. Phys. (N.Y.) 172, 304 (1986).

[45] T. Jacobson, G. Kuang, and R. Myers, Phys. Rev. D 49, 6587 (1994).

[46] M. Visser, Phys. Rev. D 48, 5697 (1993).

[47] M. B. Green, J. H. Schwarz, and E. Witten, Superstring theory, (Cambridge University Press, Cambridge 1987).

[48] T. Padmanabhan, Mod. Phys. Lett. A 17, 1147 (2002).

[49] S. Carlip, Classical Quantum Gravity 22, R85 (2005).

[50] D. V. Fursaev, Phys. Part. Nucl. 36, 81 (2005). 